\title{
Understanding Mobile Web and Mobile Search Use in Today's Dynamic Mobile Landscape
}

\author{
Karen Church \\ Telefonica Research \\ Torre Telefonica - Diagonal 00 \\ Pl. Ernest Lluch i Marti 5 \\ 08019 Barcelona, Spain \\ karen@tid.es
}

\author{
Nuria Oliver \\ Telefonica Research \\ Torre Telefonica - Diagonal 00 \\ Pl. Ernest Lluch i Marti 5 \\ 08019 Barcelona, Spain \\ nuriao@tid.es
}

\begin{abstract}
The term mobile $W e b$ is changing. Mobile is traditionally associated with on-the-move, portable and dynamic. However, with the advent of smartphones, an increasing number of users are accessing the mobile Internet via their phone while in more stationary and familiar settings, like at home or at work. This shift in the meaning of mobile is having a significant effect on mobile Web behavior. Designing great mobile Web experiences requires a deeper understanding of the information needs, behaviors and underlying motivations of mobile users. As such, the goal of this work is to study this shift and its impact on mobile Internet access, with a view to determining what this means for the future of the mobile Web and in particular mobile search. In this paper we present the results of an online diary and interview study of 18 active mobile Web users over a 4-week period focusing on how, why, where and in what situations people use the mobile Internet and mobile search. Our findings raise a new set of open research questions and point to a number of implications for enriching the experiences of mobile Web users.
\end{abstract}

\section{Author Keywords}

Mobile web, mobile search, mobile internet, user behavior, diary study, context, design implications

\section{ACM Classification Keywords}

H.m Information Systems: Miscellaneous

\section{General Terms \\ Design, Experimentation, Human Factors}

\section{INTRODUCTION}

Mobile phones are starting to dominate as the primary mode of accessing the Internet while on-the-move. This

Permission to make digital or hard copies of all or part of this work for personal or classroom use is granted without fee provided that copies are not made or distributed for profit or commercial advantage and that copies bear this notice and the full citation on the first page. To copy otherwise, or republish, to post on servers or to redistribute to lists, requires prior specific permission and/or a fee.

MobileHCI 2011, Aug 30-Sept 2, 2011, Stockholm, Sweden.

Copyright 2011 ACM 978-1-4503-0541-9/11/08-09....\$10.00. growth in popularity is due to a number of factors including: improved mobile broadband and mobile networks, the growing popularity of social networking, video services and VOIP services, as well as significant advances in mobile handset technology ${ }^{1}$. In the past two years, there has been a flurry of reports showcasing this growth. For example, a 2009 report by Morgan Stanley highlighted that the mobile Internet is growing faster than the desktop Internet ever $\operatorname{did}^{2}$. A study published by Nielsen in 2010 shows that mobile Internet usage is increasing significantly, in particular among young people $^{3}$. Furthermore, according to a report by Deloitte in 2011, more than 50\% of computing devices sold globally will be smartphones, tablets and non-PC netbooks, thus breaking the long-held market dominance of $\mathrm{PC}^{4}{ }^{4}$.

Mobile traditionally meant on-the-move, however, it has been shown that more and more users are accessing the mobile Web in non-mobile settings (i.e. at home sitting on the couch watching TV or while in bed)[19]. We believe that this shift will have a significant effect on mobile Web behavior and may present new challenges for researchers and practitioners. The goal of this work is twofold: (1) to explore mobile Web behaviour in 2011 and compare our findings to previous studies and (2) to shed new light on mobile search behaviour by exploring the impact of context on the nature of queries executed by mobile users. To achieve this goal we conducted a 4-week diary and interview study with 18 active mobile Internet users. Our study focuses on how, why, where and in what situations people use the mobile Internet and in particular mobile search.

\section{RELATED WORK}

We identified three strands of related research: (1) understanding mobile information needs, (2) exploring the nature and motivations surrounding mobile Web access and (3) analyzing real mobile Web usage patterns. In this section we discuss each of these areas in more detail.

\footnotetext{
${ }^{1}$ Admob, "Mobile Metrics Report", 2010 See: http://bit.ly/ b7DYFJ, last accessed Jun 2011

${ }^{2}$ Morgan Stanley, "The Mobile Internet Report", 2009 See, http://bit.1y/4JJDq4, last accessed Jun 2011

${ }^{3}$ Nielsen, "Mobile Youth Around The World", 2010 See, http://bit.ly/fzmCPK, last accessed Jun 2011

${ }^{4}$ Deloitte, "Technology Predictions 2011", 2011 See, http: //bit.1y/fesYLW, last accessed Jun 2011
} 


\section{Understanding Mobile Information Needs}

There have been a number of recent studies focusing on user-centric approaches to understanding the nature of mobile information needs. Sohn et al.[20] carried out a diary study of mobile information needs looking at the types of needs that arise while on-the-go and how users address those needs. The authors found that $72 \%$ of entries were prompted by explicit contextual factors including activity, location, time and conversation.

Church \& Smyth [4] carried out a similar study but allowed participants to track all information needs that is while at home, at work or while mobile. The majority of entries (67\%) were generated when users were away from their familiar contexts. Similarly, contexts like location, time, activity and social interactions have an affect on the type of needs that arise while mobile.

Heimonen [10] carried out a 4-week diary study focusing on experienced mobile Internet users. Mobile search and mobile browsing were the dominant form of addressing mobile information needs by this user group. The author found that search is used to satisfy broad information needs while known Web services and mobile applications were used more often for specific needs which are time constrained.

Hinze et al. [12] focus more on the influence of contexts such as location and activities on mobile information needs. The authors found that the types of needs expressed by users varied considerably across locations.

Most recently, Chua et al. [3] explore information need fulfillment via a 1 -week diary and interview study. The authors confirm that the contexts that trigger information needs are indeed location, intended activity and social surroundings, however, the factors affecting fulfillment also included time.

\section{Understanding Mobile Web Usage}

Other research looks beyond mobile information needs and towards why and how people access information via the mobile Internet. Lee et al. [17] examined when the mobile Internet is used most frequently and in what context it is most useful. Taylor et al. [22] explored the motivations of mobile Internet users in a 3-part study. Part 1 focused on 11 mobile Internet users in the U.S. over a 5-day period from which a taxonomy of mobile Web behaviours and motivations emerged. In parts 2 and 3 the authors validated their taxonomy and compared iPhone versus non-iPhone Web usage. The authors found the most frequent motivation among mobile Web users is awareness, a motivation usually satisfied with status checking behavior.

In [7], Cui \&Roto present results from a series of studies carried out from 2004-2007, ranging from contextual interviews to $\log$ analysis. Key results from the study include the fact that many users access the mobile Web in stationary situations and in short bursty sessions.
Google's location-based mobile usage is studied in [1]. The authors combine a mobile application which logs user searches and a diary tool to gather their results. One interesting finding is that location-based search is a social activity often conducted in the presence of others.

Nylander et al. [19] carried out a 1-week diary study involving 19 users focusing on where and why people access the mobile Web. The authors highlight that the most frequent location for mobile Internet access was at home $(31 \%)$, with many users choosing their phone over their computer for convenience.

Kaikkonen [13] carried out a large scale online survey along with interviews with a subset of 23 participants focusing on access patterns associated with full websites or tailored mobile versions of websites. Results show that European and American participants opted for full websites, while in Asia users tend to use mobile-specific content served through operator portals.

Finally, Hinman et al.[11] carried out a study of mobile Internet needs through a PC deprivation study. One of the key findings from this study was that people used their PC experiences and tried to port them to the mobile Web but in many cases did not succeed.

\section{Analyzing Real Mobile Usage}

Over the past 5 years, there have a number of studies that analyze the mobile Web usage patterns of real mobile subscribers. Many of these studies have focused on the mobile search behaviour of commercial mobile search engines like Google $[14,15,16]$ and Yahoo! [2, $24]$ as well as operator-specific mobile search services $[6,5,23]$. In general the findings from these studies can be summarized as follows: (1) mobile queries are short (2.3-2.6 terms on average); (2) mobile searchers tend to submit fewer queries per session compared to desktop users; (3) adult related content is very popular among mobile users; and (4) mobile queries have become less homogenous over the past few years. Some studies have found significant variations in the query patterns across different regions $[2,24]$. Other work focusing on temporal dynamics indicates that queries are executed in a bursty manner, with a large portion of users $(80 \%)$ issuing a single query per week [23].

It's interesting to note that the increased popularity of smartphones appears to be impacting mobile search behaviour. For example, the latest Google study [16] compares queries issued via desktops, iPhones and other mobile phones and suggests that desktop and iPhone users search in similar ways and for similar content.

\section{Our contributions}

This paper makes two key contributions. Firstly, our findings help validate previous results from fellow researchers using a dataset from 2011 as well as revealing evolving mobile Web behaviours when compared to previous work. Secondly, we delve deeper into the motivations, intent and impact of context surrounding mo- 
bile search. There have been many log analysis studies which report what mobile users search for $[14,15,16,2$, $24,6,5,23]$. However, little attention has been given to the contexts and motivations surrounding their mobile search behaviours. Our results help shed light on search behaviour from the users point of view.

\section{STUDY METHODOLOGY}

We conducted an online diary study with follow-up interviews with 18 participants over a 4 -week period where we asked users to share details of their mobile Web access sessions. We define mobile Web access as access to the Internet through a browser, search service, email client or native mobile application via a mobile phone. We chose a diary study because it is a longitudinal method that allows participants to self-record specific aspects of their behaviors. Instead of asking users to carry around a small notebook, users accessed an easy to use, online diary tool and we employed followup interviews to clarify entries and elaborate on their experiences. We focused on active mobile Internet users so that we could draw interesting insights from these early-adopters of mobile technologies. Note that we define active mobile Internet users as users who access the Internet on their mobile phone daily or multiple times per day via a smartphone only ${ }^{5}$.

\section{Participants}

Our participants were recruited using a small Web survey published via online blogs, Twitter and external mailing lists. The survey was also forwarded to friends and colleagues of the authors. In the survey, participants were asked a range of questions about their activities in terms of mobile Web usage and the frequency at which they engage in mobile Internet behavior. For this study, we selected a subset of 18 participants (11 male and 7 female) who actively browse, search and consume mobile Web content ${ }^{6}$.

Participants ranged in age between 19-47, with an average age of 29 (standard deviation: 7.7). Our participants came from a diverse range of backgrounds and professions including students, medical professionals, social workers, writers, engineers, sales assistants and job seekers. The majority of our participants lived in Ireland (13 participants), with 5 users living in other European countries (UK, Spain, The Netherlands and Finland). All of our users owned smartphones: 12 users owned an iPhone, ( 5 of whom have a 4 th generation iPhone); 4 users owned HTC handsets including the Desire, Wildfire and Hero; 1 user owned a Sony Ericsson and 1 user owned a Nokia handset. All of our participants had a flat-rate data plan and all of the users employed WiFi capabilities frequently.

\footnotetext{
${ }^{5}$ We focused on smartphone users to avoid any bias that may have be introduced by users of less sophisticated phones

${ }^{6}$ We actually started with 22 users (12 male, 10 female), but 4 users dropped out of the study for personal reasons. Hence, the results reported in this study are solely based on the data generated by and the interview feedback from these 18 users.
}

\section{Procedure}

Participants were asked to record their mobile Internet sessions in a Web-based diary tool made available online. The study ran for a period of four weeks in Jan 2011. The diary tool was accessible from both mobile and desktop Web-browsers and was designed to capture the motivations and intent surrounding mobile Web accesses while minimizing the time burden on the participants. To achieve this balance the survey included a mix of open-ended questions as well as closed questions where the user made selections from a given set of possible answers. Following is a list of the questions we asked each user to record along with a flag indicating whether the question was open $/ \operatorname{closed}^{7}$ :

1. Approximate date and time of mobile Internet access [closed].

2. The information need they were trying to address [open].

3. Their motivation for accessing the mobile Web [open].

4. Their location at the time of the Internet access [closed].

5. Who they were with [closed].

6. What they were doing [open].

7. The general task [closed].

8. If their need for information was important [closed].

9. If their need/task was accomplished [closed].

10. If their need/task was left unaccomplished, why was this the case? [open].

Users were asked to submit a single entry for every access session and to try to do so as accurately as possible and as close to the time of the Internet access as possible, i.e. at the time of access or soon after the access. It is worth noting that given that many users engaged in repetitive mobile Internet behaviour (e.g. checking emails or updating Facebook), we received multiple requests from users to include a facility in the online diary to "replicate" a diary entry. Therefore, we implemented and added this replication feature during the first few days of the study ${ }^{8}$. Many of the replicated entries $(>83 \%)$ relate to repetitive patterns such as morning/evening accesses to email, news applications, and social services like Twitter and Facebook.

Participants were sent a daily SMS for the duration of the study to remind them to fill in the diary. We varied the time of day at which this SMS was sent to capture a range of mobile Web accesses. We also sent a few emails (2/3 during the course of the study) to

\footnotetext{
${ }^{7}$ Note that we do not have any details related to the time/duration of their mobile Web access, however, this is something we addressed in the post-study telephone interview which we will return to in the latter part of this paper. ${ }^{8}$ The replicate feature allowed the user to replicate all information associated with a previous diary entry like the information need, their motivation, the tool used, etc. However, users needed to specify the new date/time and were asked to review the remaining details like what they were doing and who they were with to ensure the diary entry was correct.
} 
keep users informed of the study. At the end of the study, each participant took part in a 20-45 minute interview in which we clarified any issues with their diary entries and asked them a range of questions related to their recent mobile Internet and mobile search behaviors. We asked them to describe the things they use the mobile Web most frequently for, the features of the mobile Web they couldn't live without and any frustrations they have with their existing experiences. These interviews followed a semi-structured format, allowing the investigator to delve deeper into the intents and specific experiences surrounding mobile Web usage. In terms of incentives, each user was given a 25 euro voucher for taking part and we also raffled three 100 euro prizes.

\section{RESULTS}

In this section, we describe key results from our study.

\section{Basic Usage Results}

Over the course of the 4-week diary study, our participants generated a total of 835 diary entries, with an average of 46.4 per user $(\min =6, \max =114$, standard deviation $=32.8$ ). $54 \%$ of the diary entries were replicated and these replicated diary entries were generated by 16 of the 18 users. Only 98 entries (11.7\%) were generated from a mobile handset, with the remaining entries submitted via a desktop/laptop. These 98 entries were generated by 6 participants, with 1 participant submitting $100 \%$ of his/her entries via a mobile phone (62 entries in total for this user).

Figure 1 shows the total number of diary entries per participant, showing high diversity in terms of the number of diary entries submitted per user. Note that one of the disadvantages of our methodology is that generating a diary entry is a somewhat manual process and involves self-reporting by the end user. As such the volume of diary entries reported here is unlikely to reflect the exact volume of actual mobile Internet usage of our participants, but rather a lower bound on their actual usage. In the post-study interviews, participants reported omitting some activities they engaged in very frequently (i.e. checking emails and accessing social networking services) as they thought submitting actual volumes of these behaviours would be somewhat excessive. However, despite this limitation, we believe that the dataset collected as part of this study provides a useful base for analyzing the contexts and motivations surrounding the mobile Web usage of this user group.

\section{Mobile Web Tools}

To gain a better understanding of what our users use the mobile Internet for, participants were asked to classify their mobile Web usage into one of 6 tools/areas. Table 1 shows the 6 tools and highlights the distribution of diary entries according to these tools. The most popular category in our study was Social tools, such as Facebook and Twitter, accounting for close to $30 \%$ of diary entries. Another popular category was other tools or applications with $24 \%$ of diary entries. The majority of these were native mobile applications for access-

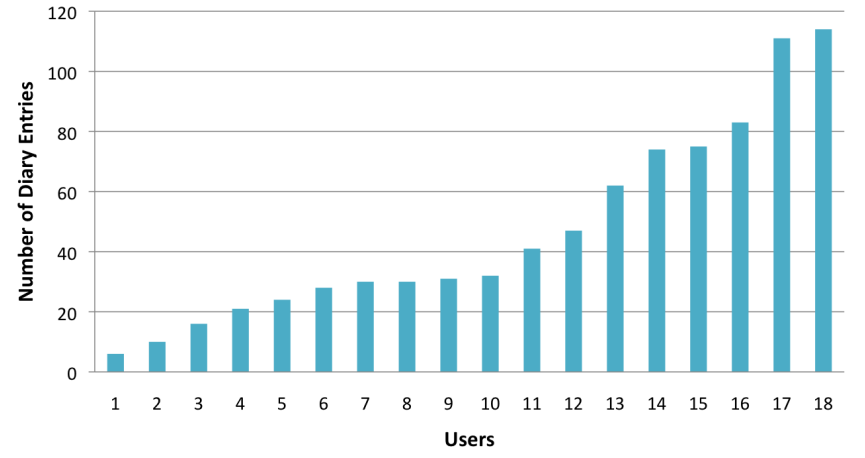

Figure 1. The distribution of number of diary entries per user (ordered by least to most frequent usage).

ing news, live music and radio services, IMBD/movie related applications, online games and general use of Android or iPhone application markets. It is interesting to note that maps and other navigation tools were not used frequently by end users (representing just 5\% of diary entries). It must be mentioned, however, that industry data shows much higher frequency of usage of mobile mapping tools[18].

\begin{tabular}{ll}
\hline Tool & \% Diary Entries \\
\hline Maps & 5.1 \\
Mobile Search & 10.0 \\
Browsing & 14.9 \\
Email & 18.8 \\
Other Tools/Apps & 24.0 \\
Social Tools/Apps & 27.2 \\
\hline
\end{tabular}

Table 1. Distribution of diary entries per tool.

In the pre-study questionnaire, most users expressed high frequency of usage of email checking (16 users indicating they checked their email multiple times per day) as well as social media websites like Facebook and Twitter (16 users doing so at least once if not multiple times per day) and given that some of these frequent behaviours were omitted from the diaries, it's likely that the percentages reported for these two categories in Table 1 would be higher in reality. Despite this, our initial results indicate a shift towards social mobile services and native mobile applications, which is inline with existing industry findings [18].

\section{The impact of context}

In this section we explore the impact of context on mobile Web behaviours.

\section{Location}

In the past, location has been considered a crucial form of context in mobile environments. However, previous work has found that mobile Web usage is increasingly occurring in static situations $[19,21]$. For example, the Nylander study [19] showed that 31\% of mobile Web usage occurs at home. Given the significant changes occurring within the mobile space, we wanted to understand if location is still a priority for today's mobile Web users and if any changes in the location of 
mobile Web accesses have emerged. As part of each diary entry, each user recorded their location at the time of their Internet access. We offered users the option to choose from one of six predefined locations ${ }^{9}$ : (1) at home, (2) at work/colleague, (3) indoors, (4) outdoors, (5) commuting/in-transit and (6) traveling abroad. Users also had the option to add an alternative location if they needed to, which we later grouped into one of the six location categories above. Table 2 shows the distribution of diary entries per location.

Probably the most interesting result is that the vast majority of diary entries (over 70\%) were recorded when users were in familiar and fairly stationary contexts, i.e. at home or at work. The volume of mobile specific contexts like traveling abroad, outdoors and commuting, represents just $17 \%$ of mobile Web usage. These results not only re-enforce earlier findings by fellow researchers $[19,21]$, but also highlight an increase in accessing the mobile Web in "stationary" situations. In follow-up interviews we confirmed that many of our users connect to online content via their mobile handset while in the comfort of their own home and engaging in everyday life like relaxing on the sofa, cooking, eating, getting ready for bed, etc. For example, one user mentioned that "Accessing the internet from my phone is easier that from my laptop because I can be on the sofa or in the bath". Another user commented that accessing the internet via his mobile allowed him "lots of 1 minute internet interactions around real life". All of the users had flat-rate data plans and the majority of our participants had WiFi in their home. Users also pointed to the fact that their mobile phones are always on and always with them thus allowing instant access to online content in an easy manner, i.e. without having to wait 5 minutes to boot up a PC/laptop. The previous Taylor et al. [22] study also found this always-on feature had a significant impact on mobile Web bheaviours. As such it appears that decreasing costs of mobile data plans, increased access to $\mathrm{WiFi}$ and the personal, always-on nature of mobile technology continue to influence stationary mobile Web usage.

\begin{tabular}{ll}
\hline Location & \% Diary Entries \\
\hline Traveling Abroad & 0.5 \\
Outdoors & 7.2 \\
Indoors & 11.4 \\
Commuting/in transit & 9.6 \\
At work/college & 21.8 \\
At home & 49.6 \\
\hline
\end{tabular}

Table 2. Distribution of diary entries per location.

\section{Social Context}

Table 3, shows the distribution of diary entries per social context. By social context we refer to whom the user was with at the time of their mobile Web usage. Note that in $>65 \%$ of cases, the user was not alone and in almost $50 \%$ of cases, participants were with someone with whom they have an intimate/close relationship, i.e. family, their partner or their friends. Our

\footnotetext{
${ }^{9}$ We based these on a subset of common locations present
} in the Nylander study[19].

\begin{tabular}{ll}
\hline Social Context & \% Diary Entries \\
\hline Other & 0.6 \\
With Strangers & 1.9 \\
With Friends & 4.8 \\
With Colleagues & 17.5 \\
With Family & 18.0 \\
With Partner & 26.0 \\
Alone & 31.3 \\
\hline
\end{tabular}

Table 3. Diary entries per social context.

discussions with participants in the post-study interviews indicate that the mobile space is being used in the presence of and shared among tightly knit social groups. For example, one user commented: "I access the Internet from my mobile to get answers quickly to group questions etc.". Another user commented that he accessed a mobile search service to search for an item because he "wanted to show a friend". Although mobile phones are personal devices, one point that became clear throughout our analysis, is that the mobile phone is primarily a communication device. It allows users to connect to the real world and interact with real people. A popular analogy made by participants was the use of the mobile Web to facilitate or enhance a conversation (particularly in our analysis of mobile search which is described later). For example, settling a friendly bet, proving someone wrong, reminiscing about past experiences, planning future events, trips or experiences, discussing an unfamiliar topic and trying to find out more information to be better informed, etc. Interestingly some users pointed to problems with this behaviour. For example, a number of users reflected on reducing their use of the phone during conversations because it resulted in their friends/family being frustrated or teasing them. Other users pointed to the fact that most of their friends had a smartphone which meant that there was always someone else within their group who could satisfy the need in question.

Both Taylor et al. [22] and Cui \& Roto [7] report on the use of the mobile Web for social facilitation (i.e. in the presence of and while engaging with others), however, Taylor et al. found that most mobile Web accesses occur while users are alone. In contrast we find much higher occurrences of social mobile Web access. As such it appears that there may be opportunity to harness the social side to mobile Web access and to provide richer online tools and mobile services to facilitate meaningful, playful and real-life interactions with family and friends.

\section{Temporal Context}

In terms of temporal context we are referring to the urgency or the importance of the task at hand, e.g. the need to find something in a hurry. As part of each diary entry, we asked users to specify if their Web access was urgent/important. We found 249 (approx. 30\%) of diary entries were flagged as urgent/important. Previous work has shown that when users are mobile, time has a significant impact on the types of needs that arise and the importance or urgency of those needs [20, 4, $10,3]$. In order to investigate if a similar trend emerges 
in mobile Web usage, we examined the volume of diary entries marked as urgent/important across the various locations specified by our participants (i.e. at home, at work, commuting, outdoors, etc. $)^{10}$. We found that when users are in mobile settings, the urgency of their needs increase. For example, when users are outdoors or commuting the urgency of their mobile Web access is between 40-47\%, while at home we find the urgency of their mobile Web access is approx. 26\%. The increased importance of mobile Web access in mobile settings was confirmed during conversations with users in the followup interviews users. Users likened this to the fact that while mobile finding a piece of information online (e.g. an address or a location) was often important to determine their next move or plans. We also found that when we focus on mobile search behaviour explicitly (discussed in later sections), the importance or urgency of the task at hand increases, indicating that search is used for more immediate needs.

\begin{tabular}{ll}
\hline Location & \% Urgent Diary Entries \\
\hline Indoors & 31.6 \\
Outdoors & 46.7 \\
Commuting/in transit & 40.0 \\
At work/college & 29.1 \\
At home & 25.6 \\
\hline
\end{tabular}

Table 4. Importance/urgency of mobile web usage according to location.

\section{Motivations}

Taylor et al. [21, 22] previously explored the motivations surrounding mobile Web access. The authors devised a list of 6 key motivators: (1) awareness, (2) time management, (3) curiosity, (4) diversion, (5) social connection and (6) social avoidance. We thought it would be interesting to investigate the key motivators for our mobile Web users and where possible, compare what we find in our study to previous results. Table 5 shows the volume and percentage of diary entries associated with each motivation. Note that we explicitly asked our users to specify their motivations while recording their diary entries. Hence, we used this motivation information along with other items in their diary entries to carry out this analysis.

\begin{tabular}{lll}
\hline Motivation & \# Diary Entries & \% Diary Entries \\
\hline Awareness & 401 & 48 \\
Time management & 205 & 24.6 \\
Curiosity & 40 & 4.8 \\
Diversion & 106 & 12.7 \\
Social Connection & 80 & 9.6 \\
Social Avoidance & 3 & 0.4 \\
\hline Total & 835 & $100 \%$ \\
\hline
\end{tabular}

Table 5. Diary entries according to motivations.

Similar to Taylor et al. [21], we find that awareness is the key motivating factor for mobile Web use, accounting for almost half of the diary entries. Tasks like checking email, checking news sites, checking twitter feeds and checking Facebook feeds where all grouped

${ }^{10}$ Note that we omitted traveling abroad from this list given that the occurrence of this location was rarely reported. into this category. Time management was also popular among our users (almost 25\% of diary entries), that is instances of mobile Web usage which enable the user to be more efficient, manage their time, plan for things, get things done, etc. Some of these relate to work or study, while others relate to finding directions, traffic reports, phone numbers, etc.

The diversion category includes any items where the user explicitly expressed their desire to kill time or alleviate boredom. We found almost $13 \%$ of mobile Web access fall into this category. In many cases users were bored at work, or were waiting on something or someone, e.g. "I was trying to pass the time while waiting for a train". Social connection is a category where the user's motivation is to connect or engage with other people, such as checking into Foursquare, updating a Facebook status, using Skype to chat with a friend, etc. Given the high use of social tools by our user group, the significant presence of this group is to be expected.

Curiosity is a category that reflects the user's interest in an unfamiliar topic which normally occurs by chance, e.g. while watching a movie and wanting more details on an actor or while chatting with a friend and wanting to prove a point. We found almost $5 \%$ of diary entries relate to this category, many of which are satisfied by using a mobile search engine or searching within a mobile specific application, e.g. IMDB movie application. This category is generally not something that is part of the user's daily routine. Instead, it is something that occurs randomly or is sparked by chance encounters, in-the-moment conversations, etc.

Finally, like the Taylor study we find that social avoidance was rare among our users. This category reflects the user's desire to stay separated from others, to appear busy, etc. We believe that the low occurrence of this motivation is not because it is rare but rather because it is likely that people are reluctant to admit that they use their mobile phone for avoidance.

\section{Temporal Patterns}

It is well known that mobile users tend to follow predictable patterns in their daily/weekly routines [8]. For example, we often wake up at similar times, work for similar hours, commute in similar ways. Previous work on general Web usage and on mobile Web navigation has shown that certain temporal patterns exist: for example, Halvey et al. [9] found that mobile browsing behavior differs based on day of the week. In order to investigate if temporal patterns have evolved, we looked at the approximate hour of mobile Internet access for each diary entry. Figure 2 shows the total percentage of diary entries per hour of the day. The graph shows two lines, one depicting the percentage of diary entries per hour of the day during weekdays only, while the other line shows this percentage during weekends only. At a high level, some recurring temporal patterns emerge. For example, we see clear peaks in the morning, around lunch time and again in the evening. By ana- 
lyzing weekends and weekdays separately, we observe a shift in the temporal patterns. For example, at weekends users appear to access the mobile Internet later in the day.

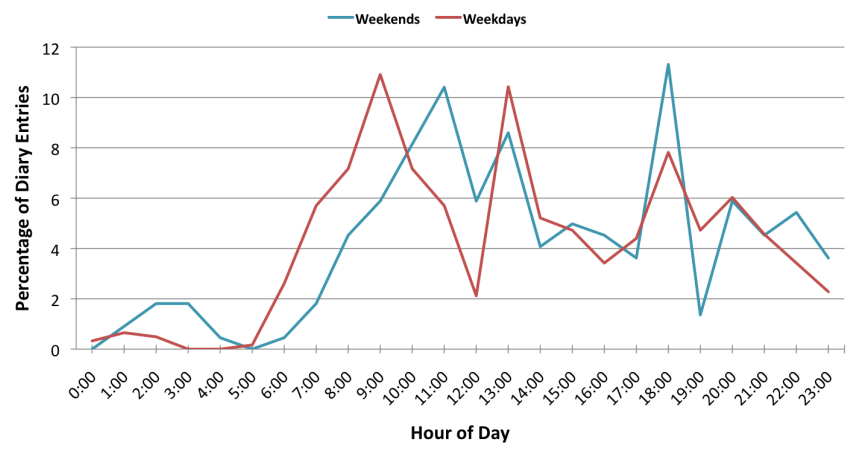

Figure 2. The temporal patterns associated with mobile web usage.

To clarify causes of these peaks, we asked each user to describe their average mobile Web usage on a typical day in our follow-up interviews. We found that time and time again, the vast majority of users describe their interactions and experiences in and around descriptions of repetitive daily routines: when $I$ wake up $I$ do $X$, when I'm getting ready to leave I do $Y$, while I'm commuting on the way to work I do Z, etc. Furthermore they tended to exhibit the same behaviours repeatedly. Many users likened this behavior to the fact that the mobile Web allowed them to maintain a connection to the outside world, accessing small nuggets of information while "living" their daily lives. There was a definite convenience factor expressed by end users in which the mobile phone allowed them to catch up on things quickly in a way that wasn't intrusive. In particular we found that this was something expressed more by users with children as they felt that it allowed them access content online without having to be away from their families or to be too distracted while enjoying some family time. Similar findings were found by Taylor et al.[22] in terms of ritual mobile Web access. Given the recurrent temporal patterns expressed by end users, in particular in stationary settings, there may be new opportunities for exploiting these repetitive behaviors in order to enhance future mobile experiences.

\section{A closer look at mobile search}

One of the key goals of our study was to try to understand the use cases, contexts, motivations and intents surrounding mobile search usage. There have been a number of recent studies which shed light on mobile query patterns and mobile search behaviors through post-task $\log$ analysis $[14,15,16,2,24,6,5,23]$. The key findings from these studies show that queries issued via average mobile phones are short (typical 2.1-2.5 terms), mobile users are interested in niche content, e.g. adult related content, mobile search sessions are short in terms of their duration and mobile search accounts for approx. $8-10 \%$ of general mobile Internet behavior.
With these points in mind we wanted to determine if we could identify any similarities between the mobile search usage exhibited by our users and the behaviors reported by these previous studies.

In terms of our study, mobile search accounts for approx. $10 \%$ of the diary entries ${ }^{11}$ which appears to be consistent with the results reported by earlier log analysis studies [6]. For each occurrence of mobile search, the participants were asked to specify the query they executed as well as details about which search engine they used. Only 3 of the 81 mobile search related diary entries correspond to the use of Yahoo!, the remaining mobile search entries correspond to the use of Google mobile search. This is also consistent with other findings on the mobile Web which highlights that Google dominates the world of mobile search[5].

We find that 17 of the 18 participants engaged in at least one mobile search session. Of the 81 occurrences of mobile search, 79 had at least one mobile query specified with an average of 3.05 terms per query $(\min =1$, $\max =9$, standard deviation $=1.48)$, and an average of 19.64 number of characters per query $(\min =7, \max =$ 59 , standard deviation $=10.37$ ). Not surprisingly our results are similar to previous work which highlights iPhone query lengths of approx. 3 terms on average [16]. This makes sense given that the vast majority of our users were iPhone or Android users. It appears that as input and interactions mechanisms on mobile handsets improve, the query lengths of mobile search will continue to increase.

In terms of contexts, we find that a significant proportion (almost $65 \%$ ) of mobile searches take place while in the presence of other people. We still find that mobile search takes place in stationary and familiar settings like home and work. However, the volume of stationary mobile search accesses is less than stationary general mobile Web access (58\% vs. 70\%). In terms of urgency or importance of the task at hand, users marked almost $60 \%$ of theirs queries as important/urgent (this compares to just $30 \%$ of urgent or important general mobile Web tasks reported previously). These results indicate that mobile search occurs in less familiar settings and tends to have more urgency or importance associated to it when compared to our previous findings on general mobile Web usage. That is mobile users use search when attempting to satisfy more immediate needs in truly mobile settings.

In terms of the topics or types of information our users searched for, we found a range of topics including travel information (e.g. bus times, etc), contact/address details of local businesses, cinema listings, recipe searches, news items and weather searches. However, we were

\footnotetext{
${ }^{11}$ We found 81 occurrences of mobile search within our dataset. Although this only represents a small sample of mobile search queries, we still feel that it is worthwhile to report on the nature of queries executed, the motivations of users and the surrounding contexts that impact on mobile search behavior.
} 
surprised by the large volume of fact checking/trivia and general informational type searches. Neither of these query types were impacted by the users location, however, both of these query types were heavily influenced by conversations or the social interactions of our participants. And exploring the motivations behind these searches helped re-enforce this point. For example, "To prove a fact I knew to other colleagues.", "I wanted to prove someone wrong.", "It came up and my son didn't know what one was", "Trying to find pictures of care bears so I could explain a tv presenter to someone.".

This social aspect to mobile search was also mentioned by users in the pre-study questionnaire and in the poststudy interview when users were asked to discuss their most recent mobile search experiences. For example, 6 of our users described their most recent mobile search sessions with some form of conversation or social interaction in mind:

User 1: "I was at dinner in a restaurant with friends and used google search and to find a band $i$ saw last year and to find out when they will play again. I used my mobile because it was readily available and could get me the answers quickly as we were talking about said band - spring break."

User 2: "I was in a restaurant talking about the Sitges film festival and the restaurant had WiFi so I looked up the festival programme for the weekend"

User 3: "Having lunch with colleagues and we couldn't remember the character name of Graham norton in $\mathrm{Fa}$ ther Ted"

User 4: "....looking for the history of the dance move The Moonwalk. I was intending to find a wikipedia page about it, and this was the first site that came up, I found the information I was looking for and proved my brother wrong about a statement he had made. We were having a discussion as to whether the moonwalk was originally a dance move or a mime theatre action..."

User 5: "The name of actor based on known film they had appeared in, I was discussing an actor in random conversation. Location was in a bar with friends socializing and I used search to fill in gaps in memory."

User 6: "Looked up imdb.com for information on a film $i$ had just watched. I was at home with friends, we all wanted to know the name of an actor, and looking it up on a phone was the fastest and easiest way to do $i t \ldots . . . "$

Given that mobile search behavior appears to be highly related to contexts like the users activity and their conversations, it is unlikely that it will follow predictable temporal patterns (as we noticed when focusing on general mobile Web usage in the earlier section). Figure 3 shows the volume of mobile search related diary entries per hour of the day. We can see mobile search behavior increasing throughout the day but we don't really notice any sharp peaks in the morning, lunch time or evenings as witnessed previously. This suggests that mobile search is not used to support repetitive daily

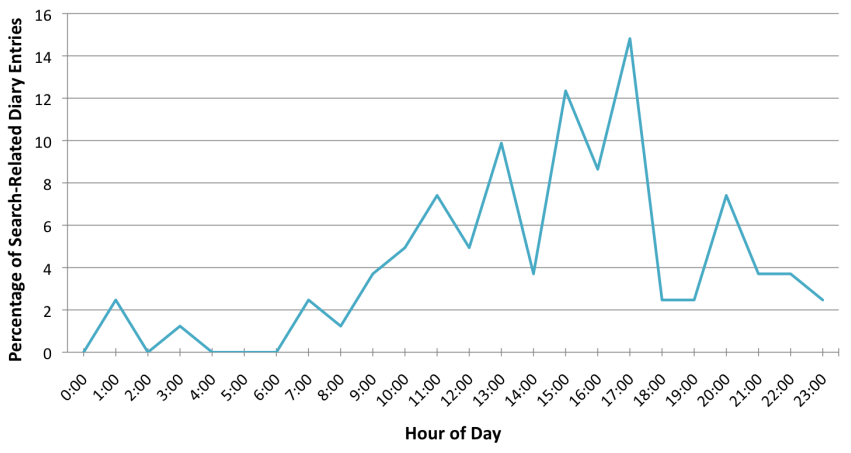

Figure 3. The temporal patterns associated with mobile search behaviour.

patterns as much as general mobile Web usage. Instead, mobile search appears to be used at random intervals to satisfy information needs that arise at that particular moment in time and such needs are heavily influenced by factors like the users activity, who they are with, the conversations they are having, etc.

Finally it is worth discussing queries with local intent. Interestingly we find that previous studies of mobile search report few occurrences of local search queries, that is queries where the user is looking for something related to a location. Despite the majority of our users having and using a maps facility, we found approx. $30 \%$ of mobile queries included some location information/location markers, e.g. "Cinema times Carlow", "things to do in San Fran", "journey planner tampere". Probing participants further helped clarify these use cases. As can be seen from the examples above, executing local searches in a general mobile search engine like Google was popular when trying to access properties of an specific event, establishment/organization or place. An explicit location was tied to the query but at a fairly high-level and the users current location may or may not have an impact. Maps, one the other hand, appear to be utilized for search when the users current location is more important.

To conclude, the results above highlight that user contexts - location and beyond - play a key role in mobile search behavior. The user's activities and chance encounters have a definite role to play. Search appears to be employed when attempting to satisfy more immediate needs. Furthermore, there is a social side to mobile search, in particular for fact-checking behaviours, that could be explored further in order to enrich mobile search experiences.

\section{Study Limitations}

A fundamental limitation of our study approach lies in the self-reported nature of diary studies. The information reported by end-users may contain some inaccuracies, it may not be complete and entries may be missing. We attempted to take some of these limitations into consideration. For example, we ensured that 
the diary could be filled from both desktop and mobile environments. We used a combination of open and closed questions to minimize the time it took to complete each diary entry. Finally, we send regular SMS and email reminders to users. Therefore, at the very least, the diary entries reported in this paper provide a realistic lower bound on mobile Web usage for our group of participants.

Another potential limitation of our study lies in our participant selection. We recruited users via forum postings, twitter re-tweets and friends-of-friends of the authors. Although our user group is not representative of the entire mobile internet population, we believe that by focusing on users with a diverse range of backgrounds and who access the mobile Web frequently, we were able to extract insights into what, how, why and when the mobile Web is used today. The dataset we gathered is significant in size compared to previous similar studies of this nature (using similar approaches). Our findings help validate findings found by fellow researchers in previous related research efforts while also helping to shed new light on emerging trends in mobile Web usage and more specifically mobile search behaviour.

\section{RESEARCH \& DESIGN OPPORTUNITIES}

The findings of our study point to a number of open research questions and design opportunities for improving the online experiences of mobile Web users. These are discussed below.

\section{Open Research Questions}

There are a number of open research questions related to general mobile Web access that we think could be explored further. Our initial results indicate that the needs and behaviours that emerge in stationary situations differ from the behaviours that emerge in real mobile settings. This is particularly true when we focused on mobile search patterns. As such it would be interesting to focuses explicitly on these differences in the future as it is likely that these behaviors will evolve. Also, given that we found some signs of recurrent daily patterns, it would be interesting to study this aspect over a longer time period and involving more users, to determine if and how such patterns could be exploited to enrich the online experiences of mobile users.

\section{Enhancing mobile search}

Our results indicate that perhaps mobile search in a traditional sense is being affected by the popularity of mobile applications. In our analysis we found $75 \%$ of mobile Web access was facilitated mainly by native mobile applications for checking email, accessing and updating social services like Facebook and Twitter, using location based services like Google maps and Foursquare as well as using news and entertainment related applications like (Youtube apps, BBC apps, IMDB apps, Radio apps, etc). Instead of searching for specific items in Google, mobile users are more likely to use a specific application for a specific purpose. For example, instead of querying for news, our users employed specific news applications. Likewise instead of issuing a query about a movie or an actor, many of our users had installed the custom IMDB application for this purpose.

Overall, users enjoyed having access to tailor-made and specific content of interest in one simply tap. From conversations with our users, it appears that one of the reasons for accessing general web content or executing a search is because the user in question has not yet found an app to fulfill his/her particular need. If we are moving towards a world of native mobile applications (at least for smartphone users), we believe that there are opportunities to facilitate users in application recommendations/suggestions. For example, searches issued though a mobile search engine reveal the user's interests in a particular topic. If a user repeatedly searches for a specific item, this might express a recurrent interest. By analyzing the mobile search behaviors of users, we might be able to use these queries to find a native application that will satisfy these needs and recommend this application to the user.

Finally we think there is scope to focus explicitly on the conversational and social aspects of mobile search. Social interactions and conversations appeared to trigger lots of fact-checking behaviours. Perhaps by studying the mobile search behaviours among close-knit social groups would reveal if and how we might exploit these social contexts to support a richer mobile search experience.

\section{CONCLUSION}

In this paper we presented the results of a diary and interview study of 18 users over a 4 week period focusing on how, why, where and in what situations people use the mobile Internet in today's evolving mobile landscape. The goal of our work was to study mobile Web access patterns in 2011, to compare results with previous findings and pinpoint evolving behaviours. We also aimed to shed new light on the contexts and motivations surrounding mobile search behaviour. Our results show that the popularity of stationary mobile web access in increasing, i.e at home or at work (over $70 \%$ of mobile web accesses). We found a preference for native mobile applications as opposed to general Web browsing. We found that the mobile Web is used in and among repetitive daily routines. However, when in mobile settings, we found a stronger impact of contexts like location, time, activity and social interactions. Finally, we found that mobile search is used in more random situations and in particular is dictated by user interactions and conversations, thus highlighting a trend towards social mobile search. Our findings point to a number of open research questions as well as potential opportunities for enriching the future experiences of mobile Web users.

As part of future work, we would like to explore demographic differences in mobile Web usage. We think there is lots of scope for additional research into the intentions and social contexts surrounding mobile search behaviours. Furthermore, we think it is important to 
continue to examine evolving mobile Web behaviours in the coming years, to determine if new trends and behaviors emerge.

\section{ACKNOWLEDGEMENTS}

This work is funded as part of a Marie Curie Intra European Fellowship for Career Development (IEF) award held by Karen Church. We would like to thank all the participants for taking part in our user study. And finally, we would like to thank the Mobile HCI reviewers and PC members for their feedback and suggestions which we believe helped to improve the paper.

\section{REFERENCES}

1. Amin, A., Townsend, S., Ossenbruggen, J., and Hardman, L. Fancy a drink in canary wharf?: A user study on location-based mobile search. In Proceedings of INTERACT '09:, Springer-Verlag (2009), 736-749.

2. Baeza-Yates, R., and Velasco, J. A study of mobile search queries in japan. In Query Log Analysis: Social and Technological Challenges, WWW 2007 Workshop (2007).

3. Chua, A. Y. K., Balkunje, R. S., and Goh, D. H.-L. Fulfilling mobile information needs: a study on the use of mobile phones. In Proceedings of ICUIMC '11, ACM (2011), 92:1-92:7.

4. Church, K., and Smyth, B. Understanding the intent behind mobile information needs. In Proceedings of IUI'09, ACM (2009), 247-256.

5. Church, K., Smyth, B., Bradley, K., and Cotter, P. A large scale study of european mobile search behaviour. In Proceedings of MobileHCI '08, ACM (2008), 13-22.

6. Church, K., Smyth, B., Cotter, P., and Bradley, K. Mobile information access: A study of emerging search behavior on the mobile internet. $A C M$ Transactions on the Web 1, 1 (2007), 4.

7. Cui, Y., and Roto, V. How people use the web on mobile devices. In Proceeding of $W W W$ '08, ACM (2008), 905-914.

8. Farrahi, K., and Gatica-Perez, D. What did you do today?: discovering daily routines from large-scale mobile data. In Proceeding of MM'08, ACM (2008), 849-852.

9. Halvey, M., Keane, M. T., and Smyth, B. Predicting navigation patterns on the mobile-internet using time of the week. In Proceedings of $W W W$ '05, ACM (2005), 958-959.

10. Heimonen, T. Information needs and practices of active mobile internet users. In Proceedings of Mobility '09, ACM (2009), 1-8.

11. Hinman, R., Spasojevic, M., and Isomursu, P. They call it surfing for a reason: identifying mobile internet needs through pc internet deprivation. In Proceedings of $\mathrm{CHI}$ '08 extended abstracts, CHI '08, ACM (2008), 2195-2208.

12. Hinze, A. M., Chang, C., and Nichols, D. M. Contextual queries express mobile information needs. In Proceedings of MobileHCI '10, ACM (2010), 327-336.

13. Kaikkonen, A. Full or tailored mobile web- where and how do people browse on their mobiles? In Proceedings of Mobility'08, ACM (2008), 28:1-28:8.

14. Kamvar, M., and Baluja, S. A large scale study of wireless search behavior: Google mobile search. In Proceedings of CHI'06, ACM (2006), 701-709.

15. Kamvar, M., and Baluja, S. Deciphering trends in mobile search. Computer 40, 8 (2007), 58-62.

16. Kamvar, M., Kellar, M., Patel, R., and Xu, Y. Computers and iphones and mobile phones, oh my!: a logs-based comparison of search users on different devices. In Proceedings of $W W W^{\prime} 09$, ACM (2009), 801-810.

17. Lee, I., Kim, J., and Kim, J. Use contexts for the mobile internet: A longitudinal study monitoring actual use of mobile internet services. International Journal of Human-Computer Interaction 18, 3 (2005), 269-292.

18. Nielsen. The state of mobile apps, 2010. See: http://bit.ly/9gSvk4.

19. Nylander, S., Lundquist, T., and Brännström, A. At home and with computer access: why and where people use cell phones to access the internet. In Proceedings of CHI'09, ACM (2009), 1639-1642.

20. Sohn, T., Li, K. A., Griswold, W. G., and Hollan, J. D. A diary study of mobile information needs. In Proceedings of CHI'08, ACM (2008), 433-442.

21. Taylor, C. A., Anicello, O., Somohano, S., Samuels, N., Whitaker, L., and Ramey, J. A. A framework for understanding mobile internet motivations and behaviors. In Proceedings of CHI'08 extended abstracts, ACM (2008), 2679-2684.

22. Taylor, C. A., Samuels, N., and Ramey, J. A. Always on: A framework for understanding personal mobile web motivations, behaviors, and contexts of use. International Journal of Mobile Human Computer Interaction 1, 4 (2009), 24-41.

23. Vojnovic, M. On mobile user behaviour patterns. In International Zurich Seminar on Communications, IEEE Communications Society (2008).

24. Yi, J., Maghoul, F., and Pedersen, J. Deciphering mobile search patterns: a study of yahoo! mobile search queries. In Proceedings of $W W W^{\prime} 08, \mathrm{ACM}$ (2008), 257-266. 